\title{
HAUSDORFF DIMENSION OF WILD FRACTALS
}

\author{
T. B. RUSHING
}

\begin{abstract}
We show that for every $s \in[n-2, n]$ there exists a homogeneously embedded wild Cantor set $C^{s}$ in $\mathbb{R}^{n}, n \geq 3$, of (local) Hausdorff dimension $s$. Also, it is shown that for every $s \in[n-2, n]$ and for any integer $k \neq n$ such that $1 \leq k \leq s$, there exist everywhere wild $k$-spheres and $k$-cells, in $\mathbb{R}^{n}, n \geq 3$, of (local) Hausdorff dimension $s$.
\end{abstract}

\section{INTRODUCTION}

Wild fractals of all achievable Hausdorff dimensions will be constructed in this paper. Since every compact metric space contains a Cantor set with the same Hausdorff dimension as that possessed by it, one is led toward attempting to realize "wild" Cantor sets of all possible Hausdorff dimensions. Specifically, the fundamental result herein asserts that for $s$ in the range $n-2 \leq s \leq n$, there is a homogeneously embedded wild Cantor set $C^{s}$ in $\mathbb{R}^{n}, n \geq 3$, of (local) Hausdorff dimension $s$. In fact, the technique of construction produces an uncountable number of such Cantor sets which are inequivalently embedded.

For $n=3, C^{s}$ will result via a carefully geometrically controlled "Antoine" construction. It will be interesting to observe that at precisely the point $s=1$, the "Antoine chains" must break apart and consequently they yield wild Cantor sets for $1<s<3$ and tame Cantor sets for $s<1$. (The cases $s=1,3$ must be handled more carefully.)

Preliminary to establishing the desired properties of fractals constructed herein, we give an adaptation of Hutchinson's [15] study of compacta defined by finite sets of contraction maps and then generalize it to compacta defined by sequences of finite sets of contraction maps. (See §2.)

It is relatively easy to show that for any $s$ such that $0 \leq s \leq n$, there is a tame Cantor set in $\mathbb{R}^{n}$ of Hausdorff dimension $s$. (See the end of $\S 1$ and $\S 2$.) On the other hand, to see that wild Cantor sets in $\mathbb{R}^{n}$ can have Hausdorff dimension only in the range codimension two and less is a more delicate issue. To see this, one invokes the notion of demension (embedding dimension) formalized by Štan'ko [27] and work of Luukkainen and Väisälä [28], [18]. We will describe this motivational background material in the next section.

My thanks to Bob Daverman for his pointed questions concerning "efficiency" (see Observation 3) which resulted in an expansion of the explanation. Thanks

Received by the editors May 12, 1990.

1980 Mathematics Subject Classification (1985 Revision). Primary 54F45, 57M30; Secondary 28A75, S4F35, 57N35.

Key words and phrases. Hausdorff dimension, Cantor set, fractal, wild, demension, similitude. 
to Henryk Torunczyk who discovered a mistake in the "proof" of Theorem 4 and made a helpful suggestion toward correcting it. Thanks to Andrejs Treibergs for programming assistance in producing Figure 1. Thanks to James Keesling for many conversations about this paper and related topics. I particularly thank James Keesling for his suggestions concerning the proofs of Theorems 2 and 4. Finally, I thank the referee for a number of suggestions which improved the exposition of this paper and to the Institute for Advanced Study where the bulk of this research was conducted.

\section{Preliminaries}

Before further discussing the main result, we shall give some useful definitions and present some background results. A Cantor set in $\mathbb{R}^{n}$ is said to be tame if there is a space homeomorphism that carries it onto the standard "middle thirds" Cantor set. Otherwise, the Cantor set is said to be wild. Examples of wild Cantor sets are given in $\mathbb{R}^{3}$ by Antoine [1] and in higher dimensions by Blankenship [3]. Perhaps the first major result on the relationship of the 1-LC property to tameness was given by Bing [2] and Homma [14] in showing that a Cantor set in $\mathbb{R}^{3}$ is tame if and only if its complement is 1-LC at every point of the Cantor set. The analogous result for $\mathbb{R}^{n}, n \geq 5$, was established by McMillan [22] and for $n=4$ by Freedman [12]. Thus, nowadays one often defines a Cantor set to be tame if its complement is 1-LC at each of its points. A subset $X$ of space $Y$ is said to be locally homotopically 1-coconnected (1-LCC) if $Y-X$ is 1-LC at all points of $X$.

There was a time when the problem of showing 1-LCC implied tameness for embeddings of polyhedra in various codimensions received considerable attention. Bryant $[4,5]$ showed that a fundamental property (the "general position prc.perty") which had been shown to hold for 1-LCC embeddings of polyhedra also held for 1-LCC embeddings of compacta. Štan'ko [27] formalized this property and equivalents and coined the term demension which is also called embedding dimension. An excellent presentation of demension theory was given by Bob Edwards [8]. There are various ways to formulate the notion of demension. Our preference is to define the demension of a compactum $X \subset \mathbb{R}^{n}(\operatorname{dem} X)$ to be $k$ if $X$ can be general positioned with respect to polyhedra in $\mathbb{R}^{n}$ the same as if $X$ were a $k$-dimensional polyhedron. Specifically, $\operatorname{dem} X \leq k$ if and only if for any closed polyhedron $L$ in $\mathbb{R}^{n}$ with $\operatorname{dim} L \leq n-k-1$, there is an arbitrarily small ambient isotopy of $\mathbb{R}^{n}$, with support arbitrarily close to $X \cap L$, which moves $L$ off of $X$.

We are particularly interested in the following basic theorem of demension theory which relates demension to covering dimension, denoted by dimension or dim. (A space $X \subset \mathbb{R}^{n}$ has covering dimension, or topological dimension, $\leq n$ if and only if every open covering has a refinement of order $\leq n$. See [16, Chapter 5].) All but one case of this theorem was proved by Bryant $[4,5]$ and Štan'ko [27]. We refer the reader to Theorem 1.4 of Edwards [8]. For $n=4$, part (2) follows from Freedman [12].

Dem/Dim Theorem. For any compactum $X \subset \mathbb{R}^{n}$,

(1) if $\operatorname{dim} X \geq n-2$, then $\operatorname{dem} X=\operatorname{dim} X$ unless $n=3$.

(2) if $\operatorname{dim} X \leq n-3$, then $\operatorname{dem} X=\operatorname{dim} X$ if an only if $X$ is 1-LCC in $\mathbb{R}^{n} ;$ otherwise, $\operatorname{dem} X=n-2$. 
For definitions of the $\alpha$-Hausdorff measure of a set $X \subset \mathbb{R}^{n}$, denoted $m_{\alpha}(X)$, and the Hausdorff dimension of $X$, denoted $\operatorname{dim}_{H} X$, see Hurewicz and Wallman [16, pp. 103 and 107, respectively]. A fractal is a space whose Hausdorff dimension is greater than its covering dimension. (See [19].) We define the topological Hausdorff dimension of $X \subset \mathbb{R}^{n}$, denoted top $\operatorname{dim}_{H} X$, to be

$$
\inf \left\{\operatorname{dim}_{H} h(X): h \text { is a homeomorphism of } \mathbb{R}^{n}\right\} .
$$

(We will see shortly that this inf can always be realized.) A set $X \subset \mathbb{R}^{n}$ is a topological fractal if its topological Hausdorff dimension is greater than its covering dimension. The following result $[28,18]$ is key motivation for this paper.

Väisälä Theorem. Let $X \subset \mathbb{R}^{n}$ be a compactum. Then, $\operatorname{dem} X \leq \operatorname{dim}_{H} h(X)$ for all homeomorphisms $h: \mathbb{R}^{n} \rightarrow \mathbb{R}^{n}$, and $\operatorname{dem} X=\operatorname{dim}_{H} h(X)$ for some $h$.

The following two propositions follow from the Dem/Dim Theorem and the Väisälä Theorem.

Proposition 1. If $X \subset \mathbb{R}^{n}$ is 1-LCC, then top $\operatorname{dim}_{H} X=\operatorname{dim} X$.

Proposition 2. If $X \subset \mathbb{R}^{n}$ is not 1-LCC, then $\operatorname{top} \operatorname{dim}_{H} X=\max (\operatorname{dim} X, n-2)$.

Let $X \subset \mathbb{R}^{n}$ be a compactum with $\operatorname{dim} X \leq n-3$, i.e., $X$ has codim 3 or greater. We say that $X$ is wild if $X$ is not $1-\mathrm{LCC}$. In this terminology, we have the following consequence of Propositions 1 and 2.

Corollary. A compactum $X \subset \mathbb{R}^{n}$ is a topological fractal if and only if it is wild and of codimension at least three.

Inside of every compact metric space with positive Hausdorff dimension, there is a Cantor set with the same Hausdorff dimension. (See Theorem 6.3 of Keesling [17].) Thus, a general philosophy is that if a counterexample or theorem exists in the theory of Hausdorff dimension, then one can be found for Cantor sets. Consequently, we are led to look at Cantor sets. In particular, any topological fractal will contain a Cantor set which is a topological fractal. Conversely, if we are given a Cantor set which is a topological fractal, we might well be able to obtain other topological fractals from it.

The corollary assures that the Cantor sets which are topological fractals are precisely the wild Cantor sets in $\mathbb{R}^{n}, n \geq 3$. In particular, any wild Cantor set $C \subset \mathbb{R}^{n}$ has Hausdorff dimension in the interval $[n-2, n]$ and there is a homeomorphism of $\mathbb{R}^{n}$ which carries $C$ to a Cantor set of Hausdorff dimension $n-2$. It is relatively easy to construct a tame Cantor set in $\mathbb{R}^{n}$ with Hausdorff dimension any preassigned element of $[0, n]$. (See the Example at the end of $\S 2$.$) With this background, it is a natural question whether for any element of$ $[n-2, n]$ one can construct a wild Cantor set in $\mathbb{R}^{n}$ with it as its Hausdorff dimension. This will be the fundamental construction of the paper.

Note that the wild Cantor sets which we construct are in a sense self-similar. A compact set $X \subset \mathbb{R}^{n}$ is said to be homogeneously embedded if for any $x, y \in$ $X$ there exists a homeomorphism $h: \mathbb{R}^{n} \rightarrow \mathbb{R}^{n}$ such that $h(x)=y$ and $h(X)=X$.

At this point, we need to hasten to add that really we are interested in producing spaces with local Hausdorf dimension correct rather than just correct "global" Hausdorff dimension. This will prevent various anomalies. If 
$x \in X \subset \mathbb{R}^{n}$, then the Hausdorff dimension at $x$ is $s$ if there are arbitrarily small neighborhoods of $x$ in $X$ of Hausdorff dimension $s$.

The idea of allowing some freedom in the construction of various topological objects and the corresponding geometric measure theoretic analysis has roots in Hausdorff's original paper [13]. In particular, he constructs Cantor sets of Hausdorff dimension $s$ for $0 \leq s \leq n$. He also constructs Jordan curves in the plane of Hausdorff dimension $s$ for $1<s<2$. He mentions that the case $s=2$ was already known.

We conclude this section by mentioning a few other related references. In [21] Mauldin and Williams gave random constructions of Sierpinski and Menger curves and calculated the Hausdorff dimension of such curves. Mauldin and Ulam [20] proposed randomly constructing various topological objects. In particular (p. 313), they raised the question of whether one particular method produced wild Cantor sets. The method of this paper differs from theirs and guarantees wildness. Falconer [9, 10] gave constructions of the solenoid and calculated the Hausdorff dimension of such objects.

\section{SiMILARITY DIMENSION AND DEFINING SEQUENCES}

Let $X$ be a metric space. If $f: X \rightarrow X$, then the Lipschitz constant of $f$ is

$$
\text { Lip } f=\sup _{x \neq y} \frac{d(f(x), f(y))}{d(x, y)} \text {. }
$$

The map $f$ is called Lipschitz if Lip $f<\infty$ and $f$ is a contraction map if Lip $f<1$. A map $S_{*}: X \rightarrow X$ is a similitude if there is a fixed $r<1$ such that

$$
d\left(S_{*}(x), S_{*}(y)\right)=r d(x, y)
$$

for all $x, y \in X$. Then $\operatorname{Lip} S_{*}=r$.

Let $S=\left\{S_{1}, \ldots, S_{n}\right\}$ be a collection of similitudes of a separable metric space $X$. Then the invariant set of the collection is the unique compact set $K$ such that $K=\bigcup_{i=1}^{n} S_{i}(K)$. (See Hutchinson [15].) The set $K$ is denoted by $|S|$.

Let $S=\left\{S_{1}, \ldots, S_{n}\right\}$ be similitudes of some Euclidean space. Let Lip $S_{i}=$ $r_{i}$. The unique positive number $D$ for which $\sum_{i=1}^{n} r_{i}{ }^{D}=1$ is called the similarity dimension of $S$.

Separation Condition. $S=\left\{S_{1}, \ldots, S_{n}\right\}$ satisfies the open set condition if there exists a nonempty open set $U$ such that

(i) $\bigcup_{i=1}^{n} S_{i}(U) \subset U$, and

(ii) $S_{i}(U) \cap S_{j}(U)=\varnothing$ if $i \neq j$.

The following theorem was proved by Moran [23]. See Hutchinson [15].

Moran Theorem. If $S=\left\{S_{1}, \ldots, S_{n}\right\}$ is a set of similitudes of $\mathbb{R}^{n}$ and if $S$ satisfies the open set condition, then the similarity dimension, $D$, of $S$ is equal to the Hausdorff dimension of $|S|$. Moreover, $0<m_{D}(|S|)<\infty$.

Not only did Moran obtain the above formula for the defining equation for the Hausdorff dimension of the generated set $|S|$, but he proved that his formula was correct by showing $0<m_{D}(|S|)<\infty$. This is a much stronger result than simply showing that the Hausdorff dimension of $|S|$ is $D$. 
Definition. A compact set $K$ in a separable metric space $X$ is said to be defined by a compact set $T$ with respect to a finite collection, $S=\left\{S_{1}, \ldots, S_{n}\right\}$ of similitudes of $X$ if

(1) $S_{i}(T) \subset T, i=1, \ldots, n$, and

(2) $K=\bigcap_{i=1}^{\infty} \cup\left\{S_{n_{1}} S_{n_{2}} \ldots S_{n_{i}}(T): n_{k} \in\{1,2, \ldots, n\}, k=1,2, \ldots, i\right\}$.

We may state (2) above a bit more succinctly in notation of Hutchinson [1] as $K=\bigcap_{i=1}^{\infty} S^{i}(T)$, where $S(T)=\bigcup_{j=1}^{n} S_{j}(T)$ and $S^{i}(T)=S\left(S^{i-1}(T)\right)$.

Definition. We call the sequence $\left\{S^{i}(T)\right\}_{i=1}^{\infty}$ above a defining sequence for $K$.

Proposition 3. If a compact set $K$ in a separable metric space $X$ is defined by a compact set $T$ with respect to a finite collection $S=\left\{S_{1}, S_{2}, \ldots, S_{n}\right\}$ of similitudes, then $K$ is the invariant set of $S$.

Proof. The lemma follows from (1), $§ 1$ of Hutchinson [15], since the nested sequence $\left\{S^{i}(T)\right\}_{i=1}^{\infty}$ converges in the Hausdorff metric to the unique limit $\bigcap_{i=1}^{\infty} S^{i}(T)$.

Proposition 4. Suppose that the compact set $K \subset \mathbb{R}^{n}, n \geq 1$, is defined by a compact set $T$ (which is the closure of the open set int $T$ ) with respect to a finite collection $S=\left\{S_{1}, \ldots, S_{n}\right\}$ of similitudes each with Lipschitz number $t<1$. Furthermore, assume that $S_{i}($ int $T) \cap S_{j}($ int $T)=\varnothing$ for $i \neq j$. Then, the Hausdorff dimension of $K$ is $-\ln n / \ln t$, i.e., $\operatorname{dim}_{H} K=-\ln n / \ln t$.

Proof of Proposition 4. By Proposition 3, we know that $K$ is the invariant set of $S=\left\{S_{1}, S_{2}, \ldots, S_{n}\right\}$. Since the Lipschitz number of each $S_{i}$ is $t$, the similarity dimension of $S$ is the number $s$ such that $n t^{s}=1$. But then $s=-\ln n / \ln t$. By hypothesis $S$ satisfies the open set condition, since $\bigcup_{i=1}^{n} S_{i}($ int $T) \subset$ int $T$ and $S_{i}($ int $T) \cap S_{j}($ int $T)=\varnothing$ if $i \neq j$. Consequently, by the Moran Theorem $\operatorname{dim}_{H} K=s$. Hence $\operatorname{dim}_{H} K=-\ln n / \ln t$ as desired.

Definition. Let $\mathscr{S}_{i}=\left\{S_{i 1}, S_{i 2}, \ldots, S_{i n_{i}}\right\}, i=1,2, \ldots$, denote a sequence of finite collections of similitudes of a separable metric space $X$. A compact set $K \subset X$ is said to be defined by a compact set $T \subset X$ with respect to $\left\{\mathscr{I}_{i}\right\}$ if

(1) $S_{i j}(T) \subset T, i=1,2, \ldots ; j=1,2, \ldots, n_{i}$, and

(2) $K=\bigcap_{i=1}^{\infty} \mathscr{S}^{i}(T)$ where $\mathscr{S}_{i}(T)=\bigcup_{j=1}^{n_{i}} S_{i j}(T)$ and $\mathscr{S}^{i}(T)=\mathscr{S}_{1} \mathscr{S}_{2} \ldots$ $\mathscr{S}_{i}(T)$.

Proposition 5. Suppose that the compact set $K$ is defined by a compact set $T \subset$ $\mathbb{R}^{n}$ with respect to a sequence $\mathscr{S}_{i}=\left\{S_{i 1}, S_{i 2}, \ldots, S_{i n_{i}}\right\}, i=1,2, \ldots$, of finite collections of similitudes. If for some constant $c>0$, we have the Lebesque measure of $\bigcup_{j=1}^{n_{i}} S_{i j}(T)$ greater than $c$ for $i=1,2, \ldots$, then $\operatorname{dim}_{H} K=n$.

Proof. Proposition 5 follows immediately from the definition of Hausdorff dimension and the fact that for $K \subset \mathbb{R}^{n}$

$$
m_{n}(K)=\frac{1}{\mathrm{Vol}_{n}} \cdot(\text { Lebesgue outer measure of } K)
$$

where $\mathrm{Vol}_{n}$ is the $n$-dimensional volume of the ball of diameter one. 
Proposition 6. Let $K \subset \mathbb{R}^{n}$ be a compactum and let $\mathscr{U}_{i}, i=1,2, \ldots$, be a sequence of countable covers of $K$ where mesh $\mathscr{U}_{i} \rightarrow 0$ as $i \rightarrow \infty$. Denote

$$
\sum_{U_{j} \in \mathscr{U}_{i}}\left(\operatorname{diam} U_{j}\right)^{p}=r_{p, i} .
$$

If for fixed $k>0, r_{p, i} \rightarrow 0$ as $i \rightarrow \infty$ for all $p>k$, then $\operatorname{dim}_{H} K \leq k$.

Proposition 6 follows immediately from the definition of Hausdorff dimension.

Example 1. In order to motivate the procedure of proof of our main result, we shall use the material of this section to establish the folklore result that for $s$ such that $0 \leq s \leq n$ there is a tame Cantor set $C^{s} \subset \mathbb{R}^{n}$ of (local) Hausdorff dimension $s$.

Proofs of this result can be found in almost any substantial work on the subject, e.g., [7, 9, 24] or even Hausdorff's paper [13].

First choose $s \in(0,1)$. Let $C^{s} \subset \mathbb{R}^{1}$ be the compact set defined by $T=$ $[0,1]$ with respect to the following two similitudes $S_{1}$ and $S_{2}$ with Lipschitz number $t=2^{-1 / s}$. Let $S_{1}$ be the isometry of $\mathbb{R}^{1}$ which carries the interval $[0,1]$ linearly onto the interval $[0, t]$ and let $S_{2}$ similarly carry $[0,1]$ onto $[1-t, 1]$. Then, it is easy to check that Proposition 4 implies that $\operatorname{dim}_{H} C^{s}=s$.

In order to achieve $s=0$, let $t_{i} \in\left(0, \frac{1}{2}\right), i=1,2, \ldots$, be a monotonically decreasing sequence tending to 0 and, in order to achieve $s=1$, let $t_{i} \in$ $\left(0, \frac{1}{2}\right), i=1,2, \ldots$, be a monotonically increasing sequence tending to $\frac{1}{2}$. For each $t_{i}$, let $\mathscr{S}_{i}=\left\{S_{i 1}, S_{i 2}\right\}$ be the set of two similitudes of $\mathbb{R}^{1}$ with Lipschitz number $t_{i}$ constructed as above. Then the sets $C^{0}$ and $C^{1}$ defined by $[0,1]$ with respect to $\left\{\mathscr{S}_{i}\right\}$ have Hausdorff dimensions 0 and 1, respectively, by Proposition 6 and Proposition 5 .

Now for $s \in(0, n)$ we will find $C^{s} \subset \mathbb{R}^{n}$ of Hausdorff dimension $s$. This time construct as above similitudes $S_{1}$ and $S_{2}$ of $\mathbb{R}^{1}$ with Lipschitz number $(2 n)^{-1 / s}$. Now consider the set $S$ with $2 n$ elements consisting of all possible $n$-products made up of $S_{1}$ and $S_{2}$. Then each element of $S$ is a similitude of $\mathbb{R}^{n}$ with Lipschitz number $(2 n)^{-1 / s}$. It is easy to check that Proposition 4 yields a Cantor set $C^{s} \subset \mathbb{R}^{n}$ of Hausdorff dimension $s$.

By now it should be clear how to apply Proposition 6 and Proposition 5 to obtain $C^{0}, C^{n} \subset \mathbb{R}^{n}$.

\section{MAIN RESUlTS AND PROOFS}

Theorem 1. Let $s$ be such that $1<s<3$. Then there exists a homogeneously embedded wild Cantor set $C^{s} \subset \mathbb{R}^{3}$ such that $\operatorname{dim}_{H} C^{s}=s$. (In fact, the local Hausdorff dimension of $C^{s}$ is $s$.)

Proof. Proposition 4 constitutes the formal idea for approaching the proof. The wild Cantor set $C^{s}$ will result from an "Antoine" construction, see Antoine [1]. The first (round) solid torus forms $T_{1}^{1} \subset \mathbb{R}^{3}$ in the construction will have inner radius $r_{1}$ and outer radius $r_{2}$. Its core circle will lie in the $x z$-plane, centered at 0 and has radius $\left(r_{1}+r_{2}\right) / 2$. The radius of a meridional disk is $\left(r_{2}-r_{1}\right) / 2$.

There are two constraints on $r_{1}$ and $r_{2}$ :

(1) $0<r_{1}<r_{2}$, and

(2) $\frac{r_{2}}{2}<r_{1}$. 
Constraint (2) results from the fact that our construction will involve a chain of tori each link of which is similar to $T_{1}^{1}$ and (2) is necessary in order to "link" the tori into a chain. (Actually we shall see that it turns out to be unnecessary to vary $r_{1}$ and $r_{2}$ to achieve all possible Hausdorff dimensions. Hence one may for instance take $r_{1}=.75$ and $r_{2}=1$.)

The second stage of our construction will be a closed chain $C_{2}=\bigcup_{i=1}^{n_{2}} T_{i}^{2}$ of tori which is embedded in the interior of $T_{1}^{1}$ and which winds around $T_{1}^{1}$ some yet to be determined number of times. (There ultimately will be great latitude in the number of times.) The chain $C_{2}$ will be constructed so that for each $T_{i}^{2}$ there is a similitude $S_{i}$ of $\mathbb{R}^{3}$ which carries $T_{1}^{1}$ onto $T_{i}^{2}$ and such that there is a fixed Lipschitz constant $t, 0<t<1$, for all $S_{i}, i=1, \ldots, n_{2}$. The Cantor set $C^{s}$ will be the invariant set of $\left\{S_{i}\right\}$, i.e., $C^{s}=\bigcup\left\{S_{i}\left(C^{s}\right)\right\}$.

We now determine the number of links, $n_{2}$, in the chain $C_{2}$ and the Lipschitz constant $t$ for the similitudes carrying $T_{1}^{1}$ onto each link. (Let us drop the subscript 2 on $n_{2}$ and let $n$ represent the number of links in $C_{2}$.)

We will perform our construction so that the similarity dimension of the invariant set of $\left\{S_{i}\right\}$ is $s$. This will be the case if and only if $n t^{s}=1$.

We must carefully choose the number $n$ of torus links in the second chain and carefully choose their size which is determined by the Lipschitz constant $t$. Let us note that, a priori, it is not apparent that appropriate $n$ and $t$ exist. For our construction, we must be able to find a "closed" chain with each link $S_{i}\left(T_{1}^{1}\right)$ of size determined by $t$ and with exactly $n$ links which can be coiled around the interior of $T_{1}^{1}$. For given $n$ and $t$ such that $n t^{s}=1$, there may well not be enough room in the interior of $T_{1}^{1}$ to coil the corresponding chain. Thus, we must see if we can judiciously choose $n$ and $t$ such that both $n t^{s}=1$ and our construction can be accomplished.

Note that our requirement that $n t^{s}=1$ is equivalent to requiring that $n=$ $1 / t^{s}$ or that $s=-(\ln n / \ln t)$. Since each link of $C_{2}$ must lie in the interior of $T_{1}^{1}$, we have the following constraint on $t$ :

$$
0<t<\frac{r_{2}-r_{1}}{2 r_{2}} .
$$

Also, since $n$ must be a natural number, $t$ must be identified so that

$$
\frac{1}{t^{s}} \text { is a natural number. }
$$

However, as we shall now show, these constraints on $t$ are still not enough to insure we can construct $C_{2}$.

One somewhat crude indication of whether $C_{2}$ can be properly constructed within $T_{1}^{1}$ for given $n$ and $t$ satisfying the above conditions is to compare the sum of the volumes of the links in a potential $C_{2}$ with the volume of $T_{1}^{1}$. Let $r V\left(t, s, r_{1}, r_{2}\right)$ denote the function of $t, s, r_{1}, r_{2}$ which represents the ratio of the sum of the volumes of the links of a chain with $n$ links of size determined by $t$ to the volume of $T_{1}^{1}$, i.e.,

$$
r V\left(t, r_{1}, r_{2}\right)=\frac{\operatorname{Vol} C_{2}}{\operatorname{Vol} T_{1}^{1}} .
$$

By integrating to find the volume of the solid $T_{1}^{1}$ of revolution, or otherwise, one sees that

$$
\operatorname{Vol} T_{1}^{1}=\frac{1}{4} \pi^{2}\left(r_{2}-r_{1}\right)^{2}\left(r_{1}+r_{1}\right) \text {. }
$$


Similarly, one determines that

$$
\operatorname{Vol} S_{i}\left(T_{1}^{1}\right)=\frac{1}{4} \pi^{2}\left[t\left(r_{2}-r_{1}\right)\right]^{2}\left[t\left(r_{2}+r_{1}\right)\right]=\frac{1}{4} \pi^{2} t^{3}\left(r_{2}-r_{1}\right)^{2}\left(r_{2}+r_{1}\right) .
$$

Consequently,

$$
r V\left(t, s, r_{1}, r_{2}\right)=\frac{\operatorname{Vol} C_{2}}{\operatorname{Vol} T_{1}^{1}}=\frac{n\left[\frac{1}{4} \pi^{2} t^{3}\left(r_{2}-r_{1}\right)^{2}\left(r_{2}+r_{1}\right)\right]}{\frac{1}{4} \pi^{2}\left(r_{2}-r_{1}\right)^{2}\left(r_{2}+r_{1}\right)}=n t^{3}=\frac{1}{t^{s}} t^{3}=t^{3-s} \text {. }
$$

As indicated above, we see that $r V\left(t, s, r_{1}, r_{2}\right)$ is independent of $r_{1}$ and $r_{2}$ and so we write in summary:

$$
r V(t, s)=t^{3-s} .
$$

So far we have the following restrictions or $r_{1}, r_{2}$ and $t$ :

(1) $\frac{r_{2}}{2}<r_{1}<r_{2}$,

(2) $0<t<\frac{r_{2}-r_{1}}{2 r_{2}}$, and

(3) $\frac{1}{t^{s}}=n$ is a natural number.

In order to understand another restriction on $t$ corresponding to values of $s$, we make the following observation. Let $r V(t, s)$ be denoted by $r V_{s}(t)$ for a fixed $s$.

Observation 1. $\lim _{t \rightarrow 0} r V_{s}(t)=0$ if and only if $s<3$.

Of course, by their definitions, we always have $s \geq 0$ and $t>0$. The observation follows since $\lim _{t \rightarrow 0} t^{3-s}=0$ if and only if $s<3$.

We see that we cannot achieve $s=3$ from our current proposed construction, because $r V(t, 3)=1$ which means that it would be necessary for a chain (of links of one size) to be packed into $T_{1}^{1}$ so as to consume all of its volume. This is clearly impossible.

By the above, for a given $s$ such that $0 \leq s<3$ we can choose $t$ small enough to have our chain $C_{2}$ consume as small of a percentage of the volume of $T_{1}^{1}$ as we like. Now we will see for which $s$ is the range $0 \leq s<3$ we can choose $t$ small and properly pack a corresponding chain into $T_{1}^{1}$ to consume the volume specified by our chosen $s$.

In order for our construction to result in a wild Cantor set of similarity dimension $s$, it must be possible to find $t$ such that a chain with link size determined by $t$ and $n=\frac{1}{t^{s}}$ links will reach around the interior of $T_{1}^{1}$ at least once. We will next examine when this can occur. (Proposition 2 indicates that for $0 \leq s<1$ this should be impossible and for $1<s<3$ this might be possible.)

Say that $r_{1}=\frac{1}{2}-\varepsilon$ and $r_{2}=\frac{1}{2}+\varepsilon$ where $\varepsilon$ is small. Also suppose that $t$ is small, i.e., $t<2 \varepsilon$. Then the diameter of a link $S_{i}\left(T_{1}^{1}\right)$ is approximately $t$ :

$$
\operatorname{diam} S_{i}\left(T_{1}^{1}\right) \dot{\sim} t
$$

Recall that $n=\frac{1}{t^{s}}$. Thus, the length of a "straight" chain with $n$-links satisfies

$$
L(t, s)<n t=\frac{1}{t^{s}} t=t^{1-s} .
$$

(Also notice that by choosing $\varepsilon$ small enough, we may make $L(t, s)$ as close to $t^{1-s}$ as we like.) Now the circumference of the core of $T_{1}^{1}$ is $\pi$. Let us denote the approximate length $t^{1-s}$ by $L^{\prime}(t, s)$. In order for our chain to circle $T_{1}^{1}$ at least once (for small $\varepsilon$ ), it will suffice to have $L^{\prime}(t, s)=t^{1-s}>\pi$ where we are allowed to choose $t$ within our constraints. 
Observation 2. $\lim _{t \rightarrow 0} L(t, s)>\pi$ if and only if $s>1$. In fact, for $s>1$ we can make $L(t, s)$ as large as we like by choosing $t$ small enough.

Now we claim that precisely when $1<s<3$, we can identify a small corresponding $t$ and construct a "closed" chain $C_{2}$ within $T_{1}^{1}$ which winds around $T_{1}^{1}$ as many times as we like. To see the claim we must see that by winding long chains around $T_{1}^{1}$, they can be packed into the interior of $T_{1}^{1}$. We first give an imprecise description of how this can be accomplished. Recall that when we studied the function $r V$ we found that the smaller we choose $t$ for a fixed $s<3$ the less the volume of $T_{1}^{1}$ the corresponding chain required. Then it seems pretty clear that if we have chosen $t$ small enough (for a fixed $s$ ) so that it winds about $T_{1}^{1}$ many times and so that it is contained in the "inner half" of $T_{1}^{1}$, then we may link the "first" link of the chain with the "last" link and pull the resulting closed chain taunt within $T_{1}^{1}$. To see that this may in fact be done, one must show that such a winding may be accomplished in an "efficient" manner.

We now describe a precise method for the winding of $C_{2}$ within $T_{1}^{1}$. (There are various ways of approaching the winding, but we will eventually describe a fairly pleasing one.) Let $T_{\varepsilon}, \varepsilon$ small, be the closed $\varepsilon$-neighborhood in $\mathbb{R}^{3}$ of the core circle of $T_{1}^{1}$. Let $\partial T_{\varepsilon}$ denote the frontier of $T_{\varepsilon}$. Then, $\partial T_{\varepsilon}$ is homeomorphic to $S^{1} \times S^{1}$. We could use as guides various torus knots $T_{p, q}$ ( $p, q$ relatively prime) which wrap around $\partial T_{\varepsilon} p$ times in the longitudinal direction and $q$ times in the meridional direction. However for simplicity we will stick with $T_{p, 1}$ torus knots. (See Rolfsen [25, p. 53].) Now for a fixed $s$ pick $t$ very small so that the corresponding chain has length sufficient to wind many times around the core of $T_{1}^{1}$ and at the same time take up little of the volume of $T_{1}^{1}$. The total number of links will be $n=1 / t^{s}$. (Perhaps this is the best place to note that we can always arrange for $n=1 / t^{s}$ to be an integer since, although $s$ is fixed, we are allowed to vary $t$ towards zero and so may judiciously pick our small $t$ so that $1 / t^{s}$ is an integer.) Now let $p$ be the largest integer so that our chain's length lies between the length of the $T_{p, 1}$ torus knot on $\partial T_{\varepsilon}$ and the length of the $T_{p+1,1}$ torus knot on $\partial T_{\varepsilon}$. Then for some $\delta \geq \varepsilon$ the $T_{p, 1}$ torus knot on $\partial T_{\delta}$ will have length the same as that of our chain and we may attempt to tauntly wind our chain within $T_{1}^{1}$ by following that $T_{p, 1}$ torus knot.

The only possible problem with the above winding procedure is that it is conceivable that even if we chose $\partial T_{\delta}$ close to $\partial T_{1}^{1}$, still the links of our chain might be too large to accomplish any winding precisely following a torus knot. (In fact this is the case for $s>2$, but not for $s<2$, see Remark 3.) Nevertheless, since we may have chosen $t$ so that our chain takes up as little of the volume of $T_{1}^{1}$ as we please, then perhaps we may break our closed chain into a finite number of chains which when closed may "collectively" be wound as above.

At this point, we have alluded to a "packing procedure" for packing $C_{2}$ within $T_{1}^{1}$ for small $t$ corresponding to a fixed $s$ between 1 and 3 . We now will describe the packing procedure in detail. (Packing problems have been around for a long time, e.g. [6].) To see that this packing can actually be accomplished for small enough $t$ corresponding to $s$, it will suffice to see that the prescribed packings can be chosen to retain enough efficiency of volume consumption as $t$ 
tends to zero. That is,

Observation 3. For a fixed $s$, the volume of the prescribed packings of the $C_{2}$ 's exceeds a fixed percentage of the "packed volume" within $T_{1}^{1}$ as $t$ tends to zero.

Perhaps the best way to see this is through the following steps:

Step 1. Given a solid torus $T=B^{2} \times S^{1}$, one can pack concentric tori of uniform cross sectional diameter $t$ into $T$ so that the volume consumed by the various collections of tori exceeds a constant multiple of the volume of $T$ as $t$ tends to zero.

To see Step 1, start with appropriate packings of disks of diameter $t$ within $B^{2}$ and then cross with $S^{1}$.

Step 2. Given an $\varepsilon$-neighborhood $T^{*}$ of a torus knot in $\mathbb{R}^{3}$ such that $T^{*}$ is homeomorphic to $B^{2} \times S^{1}$, there exists $\delta>0$ such that one can pack concentric tori (each of which is the $\delta$-neighborhood of its cone) into $T^{*}$ so that the volume consumed by the various collections of tori exceeds a constant multiple of the volume of $T^{*}$ as $\delta$ tends to zero.

Step 3. Suppose that we are given a chain $C_{2}$ which follows a torus knot and lies in the $\varepsilon$-neighborhood $T^{*}$ of the torus knot. Suppose that $C_{2}$ consumes $x \%$ of the volume of $T^{*}$. Then, we can pack $T^{*}$ with concentric chains of link diameter $t$ so that the volume of their union exceeds a constant multiple of $x \%$ of the volume of $T^{*}$ as $t$ tends to zero.

The above procedure allows one to simply base the construction on $T_{1,0}$ torus knots. However in that case $C_{2}$ will often be composed of many concentric chains. Of course, it is intuitively plausible that we should be able to break them apart and rejoin them into a single chain which winds longitudinally around $T_{1}^{1}$. However, rigor requires a canonical description of such windings which retain enough efficiency of volume consumption. The above discussion leads to satisfactory canonical windings which follow what we shall refer to as "bedspring knots." Think of a circle of bedsprings with large ends and small ends alternating; see Figure 1.

In order to see that a sufficiently efficient chain packing based on a bedspring knot can always be accomplished, we use highly compressed bedspring knots of type $T_{1,2 q \times r}$. Such a knot winds around $T_{1}^{1}$ once in the longitudinal direction, $2 q \times r$ times in the meridional direction and has $2 q$-springs with each spring having $r$ coils. (We could also use $T_{2 p \times r, 1}$ bedspring knots which would represent the intuitive description given above, but a picture is harder to visualize.)

Given $\varepsilon>0$, we may construct an " $\varepsilon$-compressed" bedspring knot of type $T_{1,2 q \times r}$ by requiring all of the "inner coils" of each spring to lie in a meridional disk, successive inner coils to lie in meridional disks about $\varepsilon$-apart and the coils to "coil" at a distance of about $\varepsilon$. Then it is not difficult to see that we can pack $C_{2}$ within $T_{1}^{1}$ for small $t$ corresponding to a fixed $s$ between 1 and 3 by following an $\varepsilon$-compressed bedspring knot. In fact, since our previously described packings for small $t$ corresponding to $s$ can be accomplished within as small of a portion of the volume of $T_{1}^{1}$ as we like, we are safe because we can extrapolate that our construction can be accomplished. For further detail see Remark 4. 


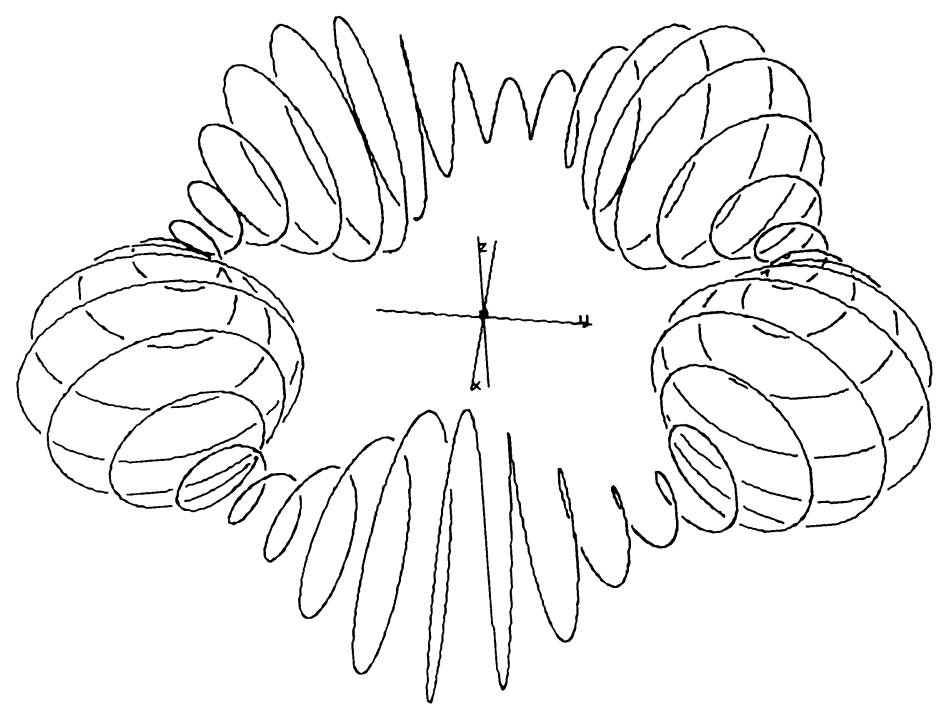

FiguRe 1

We now iterate our construction by using the same $t$ at each stage for our fixed $s$, to produce $C_{3}, C_{4}, \ldots$. The set $C^{s}=\bigcap_{i=1}^{\infty} C_{i} \quad\left(C_{1}=T_{1}^{1}\right)$ is the Cantor set we seek. In particular, by Proposition 1, it is the invariant set for the $n=1 / t^{s}$ similitudes of Lipschitz number $t$ which carry $T_{1}^{1}$ onto the respective links in the $C_{2}$ stage. Proposition 4 now applies to our construction, where $C^{s}$ corresponds to $K$ and $T_{1}^{1}$ corresponds to $T$. $C^{s}$ is clearly homogeneous since each stage of construction evolves from iterations of a single chain.

Remark 1. Note that $\pi_{1}\left(\mathbb{R}^{3}-C^{s}\right) \not \approx 0$. In fact, $C^{s}$ fails to be 1-LCC at every point and so is everywhere wild.

Remark 2. The construction for Theorem 1 also works for $s$ such that $0<s<$ 1 , however in that range the tori in $C_{2}$ are unlinked. Hence the construction yields a tame Cantor set $C^{s}$ of Hausdorff dimension $s$. In fact, for any $s$ such that $0<s<3$, we can make $C_{2}$ up of unlinked tori and obtain a tame Cantor set of Hausdorff dimension $s$. One gleans satisfying intuition for Propositions 1 and 2 by observing that "the chains must fall apart precisely at $s=1$ ".

Remark 3. It is interesting to note that the chain $C_{2}$ (and successive chains) can "follow" a single torus knot for $1<s<2$ and not for $2<s$. To see this, let us call a chain of link size determined by $t$ a " $t$-chain". The maximal length of a $t$-chain that can for instance follow a $T_{1, q}$ torus knot inside of $T_{1}$ is approximately:

$$
\tilde{L}_{t}=\left(\frac{2 \pi r_{1}}{t\left(r_{2}-r_{1}\right)}\right) \pi\left(r_{2}-r_{1}\right)=t^{-1} 2 \pi^{2} r_{1} .
$$

(Here $\tilde{L}_{t}$ represents the total length of a collection of meridional circles about $\partial T_{1}$ spaced $t\left(r_{2}-r_{1}\right)$ apart.) We have seen that for a fixed $s$ the "length" of $C_{2}$ is approximately:

$$
L^{\prime}(t, s)=t^{1-s}
$$




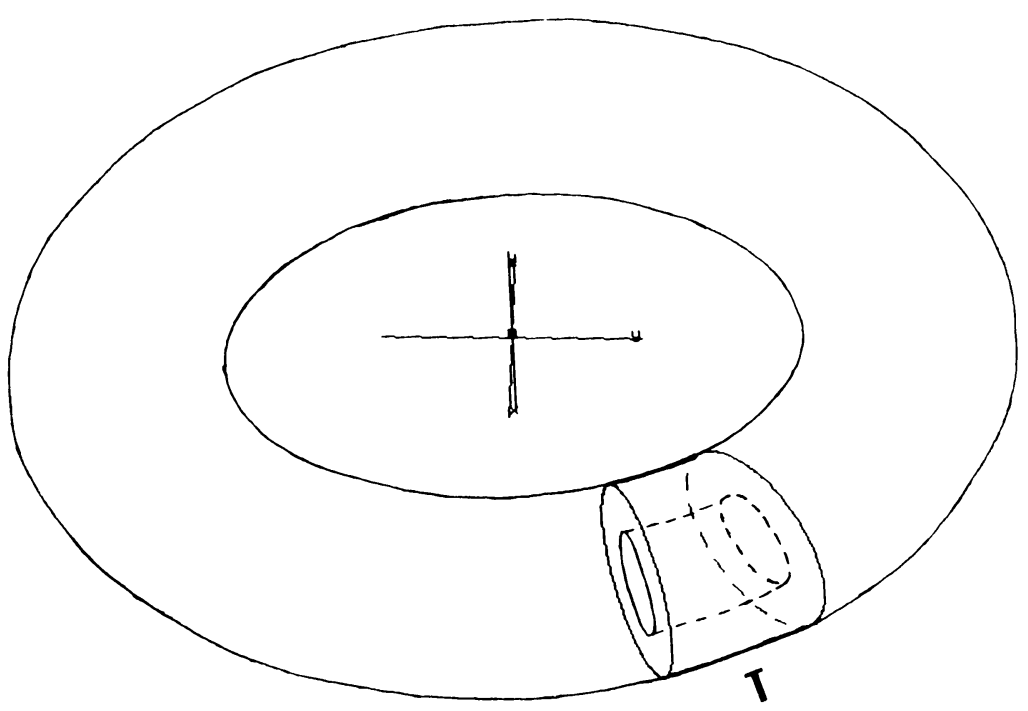

FIGURE 2

Thus,

$$
\frac{\tilde{L}_{t}}{L^{\prime}(t, s)}=\frac{t^{-1}\left(2 \pi^{2} r_{1}\right)}{t^{1-s}}=C t^{s-2}, \quad C=\text { constant }
$$

and

$$
\lim _{t \rightarrow 0} \frac{\tilde{L}_{t}}{L^{\prime}(t, s)}=\infty \quad \text { if and only if } s<2 .
$$

Remark 4. We elaborate here on why we can pack $C_{2}$ within $T_{1}^{1}$ for small $t$ corresponding to a fixed $s$ between 1 and 3 by following an $\varepsilon$-compressed bedspring knot. For convenience, we shall use "alternating" bedspring knots, i.e., the orientation of the winding reverses from spring to spring. Also, for display purposes, we coil in the meridional direction rather than the longitudinal direction; however, since the bedspring knots lie in a copy of $S^{1} \times S^{1} \times I$ one may visualize the longitudinal case by reversing the $S^{1}$ factors.

By previous arguments, we may realize $C_{2}$ (for a small $t$ corresponding to $s$ ) by concentric chains packed in $T$ (see Figure 2 ) in a vertically "laminated" fashion. Now the levels of concentric circles of chains may be slipped around $T_{1}^{1}$ so as to be evenly spaced around $T_{1}^{1}$. Each collection of concentric chains may be reformed into a spiral by unlinking each chain and relinking the first link to the last link of the "proceeding" chain, and the last link with the first link of the next chain. (At this point the first and last links of the spiral are left dangling.) Without loss of generality, we may assume that there are an even number of spirals. Now we join these spirals of chains into a single chain. We will start with a fixed spiral and move clockwise around $T_{1}^{1}$. Link the first link of the first spiral with the first link of the second spiral. Link the last link of the second spiral with the last link of the third spiral. Link the first link of the third spiral with the first link of the fourth spiral, etc. Then the resulting chain follows a compressed alternating bedspring knot as pictured in Figure 3. 


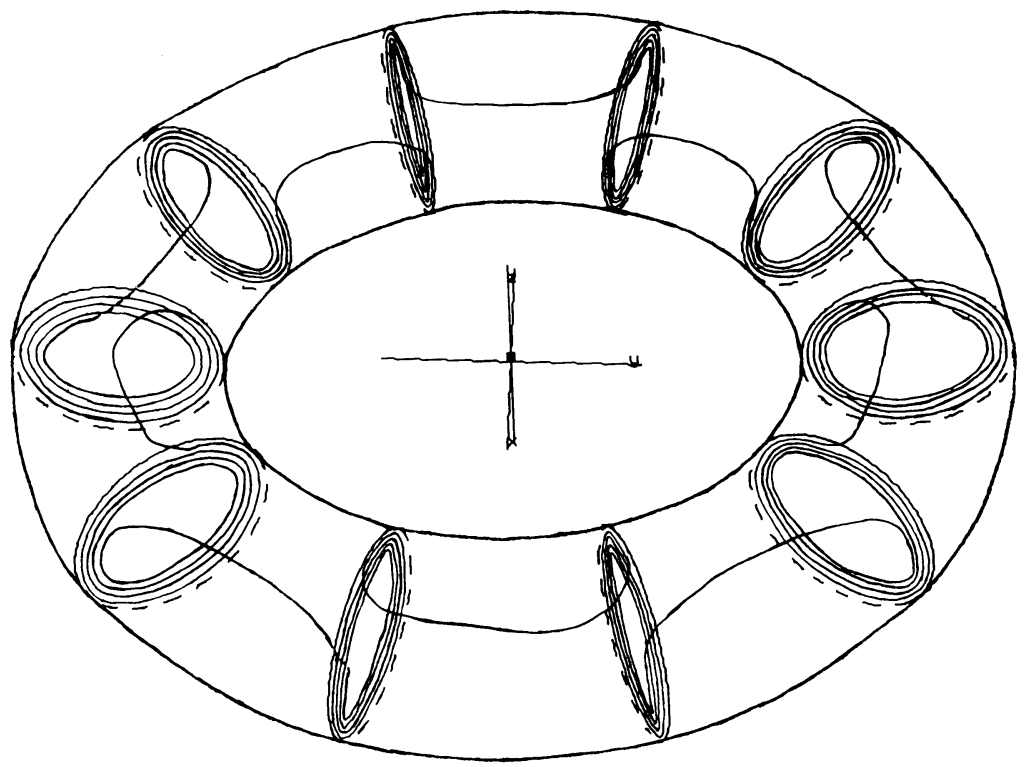

FIGURE 3

Theorem 2. There exist homogeneously embedded wild Cantor sets $C^{1}$ and $C^{3}$ in $\mathbb{R}^{3}$ such that $\operatorname{dim}_{H} C^{1}=1$ and $\operatorname{dim}_{H} C^{3}=3$. (In fact the local Hausdorff dimension of $C^{1}$ and $C^{3}$ are 1 and 3 , respectively.)

Proof. We will first do the easiest case, i.e., construct $C^{1}$. Let $s_{i}=\frac{i+1}{i}, i=$ $1,2, \ldots$. Then, $\left\{s_{i}\right\}$ is a monotonically decreasing sequence which converges to 1 . By the construction in our proof of Theorem 1 , for each $s_{i}$, we can identify a collection of similitudes $\mathscr{S}_{i}=\left\{S_{i 1}, S_{i 2}, \ldots, S_{i n_{i}}\right\}$ for which the compact set $T_{1}^{1}$ defines a Cantor set of Hausdorff dimension $s_{i}$. Let $t_{i}$ be the common Lipschitz number for this collection of similitudes. Let $C^{1}$ denote the Cantor set defined by $T_{1}^{1}$ with respect to $\left\{\mathscr{S}_{i}\right\}$. Then by Proposition 6, $\operatorname{dim}_{H} C^{1} \leq 1$. (Apply Proposition 6 where $\mathscr{U}_{i}$ is the cover of $C^{1}$ by all the links of the $i$ th stage.) But $\operatorname{dim}_{H} C^{1} \geq 1$ by Proposition 2 since $C^{1}$ is wild. Thus, $\operatorname{dim}_{H} C^{1}=1$.

We now construct $C^{3}$. Let us begin by considering an example.

Example 2. Let $s_{i}=\frac{3 i-1}{i}, i=1,2, \ldots$. Then $\left\{s_{i}\right\}$ is a monotonically increasing sequence which converges to 3 . By the construction in our proof of Theorem 1, for each $s_{i}$, we can identify a collection of similitudes $\mathscr{S}_{i}=$ $\left\{S_{i 1}, S_{i 2}, \ldots, S_{i n_{i}}\right\}$ for which the compact set $T_{1}^{1}$ defines a Cantor set of Hausdorff dimension $s_{i}$. Let $t_{i}$ be the common Lipschitz number for this collection of similitudes. Let $C^{*}$ denote the Cantor set defined by $T_{1}^{1}$ with respect to $\left\{\mathscr{S}_{i}\right\}$.

Although it seems reasonable to conjecture that $\operatorname{dim}_{H} C^{*}=3$, we have not yet developed a proof. (See Remark 5 below.) Thus, we will augment the construction of Example 2 in order to be able to apply Proposition 5 to obtain the desired $C^{3}$. For that purpose, fix some $c$ where $0<c<\operatorname{Vol} T_{1}^{1}$. First, we 
want to properly define a finite collection of similitudes

$$
\mathscr{S}_{1}^{\prime}=\left\{S_{11}^{\prime}, S_{12}^{\prime}, \ldots, S_{1 n_{1}^{\prime}}^{\prime}\right\} \text { with } \operatorname{Vol} \bigcup_{S \in \mathscr{S}_{1}^{\prime}} S\left(T_{1}^{1}\right)>c .
$$

We begin the collection $\mathscr{S}_{1}^{\prime}$ by including the collection $\mathscr{S}_{1}$ of Example 2. Unfortunately, it may not be the case that Vol $\bigcup_{S \in \mathscr{S}_{1}} S\left(T_{1}^{1}\right)>c$. Consequently, pack $T_{1}^{1}-\bigcup_{S \in \mathscr{S}_{1}} S\left(T_{1}^{1}\right)$ with a finite collection of tori $\mathscr{T}_{2}=\left\{T_{i}^{1}\right\}_{i=2}^{k_{2}}$ each with Lipschitz number much less than $t_{1}$. One can arrange that these tori do not link each other nor $T_{1}^{1}$. Now for each torus $T_{i}^{1} \in \mathscr{T}_{2}$, one may obtain a collection of $n_{1}$ similitudes $\mathscr{S}_{i}^{1}$ which collectively embed $T_{1}^{1}$ into $T_{i}^{1}$ just as the collection $\mathscr{S}_{1}$ embedded $T_{1}^{1}$ into itself. Now we have the collection $\mathscr{S}_{1} \cup\left\{\mathscr{S}_{i}^{1}\right\}_{i=2}^{k_{2}}$ of similitudes the union of whose images consume more of the volume of $T_{1}^{1}$ that did those of $S_{1}$. If $\bigcup_{S \in \mathscr{S}_{1} \cup\left\{\mathscr{S}_{i}^{1}\right\}_{i=2}^{k_{2}}} S\left(T_{1}^{1}\right)$ does not yet consume $c$ volume, then we continue to add to the collection of similitudes by packing

$$
T_{1}^{1}-\left[\bigcup_{S \in \mathscr{S}_{1} \cup\left\{S_{i}^{1}\right\}_{i=2}^{k_{2}}} S\left(T_{1}^{1}\right)\right]
$$

with even smaller collections of closed chains consisting of $n_{1}$ tori just as above. By iterating the procedure one may eventually obtain the finite collection $\mathscr{S}_{1}^{\prime}$ of similitudes whose images of $T_{1}^{1}$ consume at least $c$ volume.

To construct $\mathscr{S}_{2}^{\prime}$ one first obtains a finite collection of similitudes for each $S\left(T_{1}^{1}\right), S \in \mathscr{S}_{1}^{\prime}$, based on the collection $\mathscr{S}_{2}$ of Example 2 . Just as in the construction of $\mathscr{S}_{1}^{\prime}$, one then continues to pack $\bigcup_{S \in \mathscr{S}_{1}^{\prime}} S\left(T_{1}^{1}\right)$ with closed chains of $n_{2}$ tori based on $\mathscr{S}_{2}$ to fill $c$ volume. (Insure that each $S\left(T_{1}^{1}\right), S \in \mathscr{S}_{1}^{\prime}$, is packed identically.) Thus, by iteration one obtains the desired sequence of finite collections of similitudes $\left\{\mathscr{S}_{i}^{\prime}\right\}_{i=1}^{\infty}$. We claim that the wild Cantor set $C^{3}$ defined by $T_{1}^{1}$ with respect to $\left\{\mathscr{S}_{i}^{\prime}\right\}, i=1,2, \ldots$, is the desired one.

First, Proposition 5 insures that $\operatorname{dim}_{H} C^{3}=3$.

It remains to see that $C^{3}$ is homogeneously embedded. Choose $x, y \in C^{3}$. Then each of $x$ and $y$ is an intersection of a nested sequence of tori, one from each collection $\left\{\mathscr{S}_{i}^{\prime}\right\}_{i=1}^{\infty}$. Pick the first place where these nested sequences differ. There are two possible situations.

Firstly, the two tori may be different tori in the same closed chain. In that case one may spin that chain of tori upon itself carrying the torus identified with $x$ into that identified with $y$. This may be done leaving all other chains fixed since no chain or link links another.

Secondly, the two tori may be from different chains. In that case, the chain in which $x$ lies may be switched with the chain in which $y$ lies with the appropriate link going onto the appropriate link. This may be accomplished leaving all other chains fixed since no chain or link links another.

One now iterates this procedure based on the pairing of the defining sequences of tori for $x$ and $y$ respectively. One sees that subsequent moves have support on ever smaller open subsets of $\mathbb{R}^{3}$ since the images of $x$ are converging toward $y$. Thus, the desired limiting homeomorphism $h$ of $\mathbb{R}^{3}$ which takes $x$ to $y$ is well-defined. 
Question. What is the Hausdorff dimension of $C^{*}$ constructed in Example 2?

Remark 5. It is plausible that $\operatorname{dim}_{H} C^{*}=3$ even though $\bigcup_{S \in S_{i}} S\left(T_{1}^{1}\right), i=$ $1,2, \ldots$, do not have the efficiency of volume consumption, as $i \rightarrow \infty$, as specified in the hypothesis of Proposition 5. The proof of Theorem 1 indicates that increasing efficiency of volume consumption is not necessary to gain Hausdorff dimension arbitrarily close to three, but rather that having enough similitudes (i.e. links) at each stage will suffice. (This fact results from Observation 2 of that proof.)

Theorem 3. For every $s \in[n-2, n]$, there exists a wild Cantor set $C^{s}$ in $\mathbb{R}^{n}, n \geq 3$, of (local) Hausdorf dimension $s$. Furthermore, $C^{s}$ is homogeneously embedded in $\mathbb{R}^{n}$.

One proves Theorem 3 by applying the Blankenship construction [3] to the proofs of Theorems 1 and 2 .

Theorem 4. For any $s \in[n-2, n]$ and for any integer $k \neq n$ such that $1 \leq$ $k \leq s$, there exists an everywhere wild $k$-sphere (and $k$-cell) in $\mathbb{R}^{n}, n \geq 3$, of (local) Hausdorff dimension $s$.

Proof. We shall first establish the case $n=3$. Note that in that case $k=1$ or 2 . Let us first describe the proof for $1 \leq s<3$. One begins with a triangulated $k$-sphere (or $k$-cell) $\Sigma_{1}$, Choose a solid torus close to the barycenter of each principal simplex of $\Sigma_{1}$ and perform the construction of the proof of Theorem 1 for $1<s<3$ or Theorem 2 for $s=1$. Now by using the resulting nested sequence of chains of tori one "tubes out" the interior of each principal simplex to its associated Cantor set with $k$-dimensional tubes which are locally polyhedral modulo the Cantor set. (See Example 2.4.12 of Rushing [26].) We denote the finite union of Cantor sets in the first stage by $C_{1}^{s}$. Now subdivide $\Sigma_{1}$ off of $C_{1}^{s}$ with a triangulation of mesh less that $\frac{1}{2}$ and repeat the same procedure on principal simplexes. Thus, one obtains $\Sigma_{2}$ and $C_{2}^{s}$. Inductively, subdivide $\Sigma_{j}$ off of $\bigcup_{i=1}^{j} C_{i}^{s}$ with a triangulation of mesh less than $\frac{1}{j+1}$ and repeat the "tubing out" procedure on principal simplexes. Thus one obtains $\Sigma_{j+1}$ and $C_{j+1}^{s}$. Now consider the map $f$ of $\Sigma_{1}$ into $\mathbb{R}^{3}$ which results from infinitely repeating this procedure.

We now want to show that by being more careful in the construction of $f: \Sigma_{1} \rightarrow \mathbb{R}^{3}$ we can ensure that $f$ is an embedding and that $f\left(\Sigma_{1}\right)$ has Hausdorff dimension $s$. Firstly, it is easy to insure that $f$ is an embedding by using the Inductive Convergence Criterion. That is, if $X$ is a compactum, and if $f_{i}: X \rightarrow \mathbb{R}^{n}$ is a sequence of embeddings, then there exist $\varepsilon_{i} \rightarrow 0$ such that if $\operatorname{dist}\left(f_{i}, f_{i+1}\right)<\varepsilon_{i}$, it is the case that $f=\lim f_{i}$ is an embedding. One simply observes that in our construction above we had the freedom to choose $\Sigma_{i+1}$ as close pointwise to $\Sigma_{i}$ as we liked. (The Inductive Convergence Criterion is Theorem 6.1.2 of J. van Mill [29]. It is due to M. K. Fort [11] and has appeared in works by R. H. Bing, J. D. Anderson, and Tom Chapman, see [2].)

Secondly, we need to see that we can arrange for the Hausdorff dimension of $f\left(\Sigma_{i}\right)$ to be $s$. Certainly it is at least $s$ since $f\left(\Sigma_{i}\right)$ contains a dense set of Hausdorff dimension $s$. Now we shall show how to refine our construction to insure that is no more than $s$. Choose $\varepsilon_{i} \rightarrow 0$. By definition of Hausdorff 
dimension, choose a cover $\mathscr{U}_{1}$ of $\Sigma_{1}$ in $\mathbb{R}^{3}$ such that

$$
\sum_{U \in \mathscr{U}_{1}}(\operatorname{diam} U)^{s+\varepsilon_{1}}<1
$$

Now construct $\Sigma_{2}$ as above to be $\mathscr{U}_{1}$ close to $\Sigma_{1}$. Next choose a cover $\mathscr{U}_{2}$ of $\Sigma_{2}$ which star refines $\mathscr{U}_{1}$ and is such that

$$
\sum_{U \in \mathscr{U}_{2}}(\operatorname{diam} U)^{s+\varepsilon_{2}}<\frac{1}{2}
$$

Continue this process inductively to obtain $\Sigma_{1}, \Sigma_{2}, \ldots \rightarrow \Sigma$. Then, the Hausdorff dimension of $\Sigma$ is $\leq s$. To see this choose $a>s$. Then there exists an $i^{\prime}$ such that $s+\varepsilon_{i^{\prime}}<a$ for all $i>i^{\prime}$. Therefore,

$$
\lim _{i \rightarrow \infty}\left(\sum_{U \in \mathscr{U}_{i}}(\operatorname{diam} U)^{a}\right) \rightarrow 0
$$

This implies that the $a$-Hausdorff measure of $\Sigma$ is 0 which in turn implies that the Hausdorff dimension of $\Sigma$ is less than $a$.

The case $s=3$ is handled as above except that at stage $i$ one "implants" a finite union $C_{i}^{3-\varepsilon_{i}}$ of Cantor sets of Hausdorff dimension $3-\varepsilon_{i}$ where $\varepsilon_{i} \rightarrow 0$. Of course, in this case we do not need to worry about the Hausdorff dimension of the resulting $\Sigma$ exceeding 3 .

Now suppose that $n>3$. (The result for $k=n-1, n-2$ may be obtained through suspensions of the above construction.) The procedure in higher dimensions is similar to the one just described for $n=3$, but uses the Cantor sets of Theorem 3 in lieu of the Cantor sets of Theorems 1 and 2. The higher dimensional procedure is described in [3] in the proof of Theorem $3 \mathrm{~F}$ of that paper.

\section{REFERENCES}

1. Louis Antoine, Sur l'homeomorphisme de deux figures et de leurs voisinages, J. Math Pures Appl. 4 (1921), 221-325.

2. R. H. Bing, Tame Cantor sets in $E^{3}$, Pacific J. Math. 11 (1961), 435-446.

3. W. A. Blankenship, Generalization of a construction of Antoine, Ann. of Math. (2) 53 (1951), 276-297.

4. J. Bryant, On embeddings of compacta in euclidean space, Proc. Amer. Math. Soc. 23 (1969), 46-51.

5. __, On embeddings of 1-dimensional compacta in $E^{5}$, Duke Math J. 38 (1971), 265-270.

6. Barry Cipra, Music of the spheres, Science 251 (1991), 1028.

7. G. A. Edgar, Measure, topology, and fractal geometry, Springer-Verlag, New York, 1990.

8. R. D. Edwards, Demension theory I, Geometric topology, edited by L. C. Glaser and T. B. Rushing, Lecture Notes in Math, vol. 438, Springer-Verlag, Berlin and New York, 1975, pp. 195-211.

9. K. J. Falconer, The geometry of fractal sets, (corrected version), Cambridge Univ. Press, 1986.

10. _ Fractal geometry, Wiley, New York, 1990.

11. M. K. Fort, Homogeneity of infinite products of manifolds with boundary, Pacific J. Math 12 (1962), 879-884.

12. M. H. Freedman, The topology of 4-manifolds, J. Differential Geometry 17 (1982), 357-453. 
13. F. Hausdorff, Dimension und áusseres mass, Math Ann. 79 (1919), 157-179.

14. T. Homma, On tame embedding of 0-dimensional compact sets in $E^{3}$, Yokohama Math. J. 7 (1959), 191-195.

15. J.E. Hutchinson, Fractals and self-similarity, Indiana Univ. Math. J 30 (1981), 713-747.

16. W. Hurewicz and H. Wallman, Dimension theory, Princeton Univ. Press, Princeton, N.J., 1941.

17. James Keesling, Hausdorff dimension, Topology Proc. 2 (1986), 349-383.

18. J. Luukkainen and J. Väisälä, Elements of Lipschitz topology, Ann. Acad. Sci. Fenn Ser. AI3 (1977), 85-122.

19. B. Mandelbrot, The fractal geometry of nature, Freeman, San Francisco, Calif., 1982.

20. R. D. Mauldin and S. M. Ulam, Mathematical problems and games, Adv. in Appl. Math. 8 (1987), 281-344.

21. R. D. Mauldin and S. C. Williams, Random recursive constructions: asymptotic geometric and topological constructions, Trans. Amer. Math. Soc. 295 (1986), 325-346.

22. D. R. McMillan, Jr., Taming Cantor sets in $E^{n}$, Bull. Amer. Math. Soc. 70 (1964), 706-708.

23. P. A. P. Moran, Additive functions of intervals and Hausdorff measure, Proc. Cambridge Philos. Soc. 42 (1946), 15-23.

24. C. A. Rogers, Hausdorff measures, Cambridge Univ. Press, London, 1970.

25. Dale Rolfsen, Knots and links, Math. Lecture Series, Publish or Perish, 1976.

26. T. B. Rushing, Topological embeddings, Academic Press, 1973.

27. M. A. Štan'ko, The embedding of compacta in euclidean space, Mat. Sb. 83(125) (1970), 234-255 [=Math. USSR-Sb. 12 (1970), 234-254]. (Announcement appeared in Dokl. Akad. Nauk SSSR 86 (1969), 1269-1272 [=Soviet Math. Dokl. 10 (1969), 758-761].)

28. J. Väisälä, Demension and measure, Proc. Amer. Math. Soc. 76 (1979), 167-168.

29. J. van Mill, Infinite-dimensional topology, North-Holland, New York, 1989. 08540

School of Mathematics, The Institute for Advanced Study, Princeton, New Jersey

Current address: Department of Mathematics, University of Utah, Salt Lake City, Utah 84112

E-mail address: rushing@math.utah.edu 